\title{
Sophie Albert, «Ensemble ou par pieces». Guiron le Courtois (XIII $-\mathrm{XV}^{\mathrm{e}}$ siècles): la cohérence en question
}

\author{
Maria Colombo Timelli
}

\section{(2) OpenEdition}

\section{Journals}

\section{Édition électronique}

URL : https://journals.openedition.org/studifrancesi/3019

DOI : 10.4000/studifrancesi.3019

ISSN : 2427-5856

\section{Éditeur}

Rosenberg \& Sellier

\section{Édition imprimée}

Date de publication : 1 juillet 2013

Pagination : 435-436

ISSN : 0039-2944

\section{Référence électronique}

Maria Colombo Timelli, «Sophie Albert, «Ensemble ou par pieces». Guiron le Courtois (xII"-xve siècles): la cohérence en question », Studi Francesi [En ligne], 170 (LVII | II) | 2013, mis en ligne le 30 novembre 2015, consulté le 02 février 2023. URL : http://journals.openedition.org/studifrancesi/3019 ; DOI : https://doi.org/10.4000/studifrancesi.3019

Ce document a été généré automatiquement le 2 février 2023

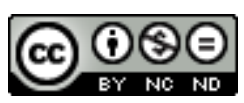

Creative Commons - Attribution - Pas d'Utilisation Commerciale - Pas de Modification 4.0 International - CC BY-NC-ND 4.0

https://creativecommons.org/licenses/by-nc-nd/4.0/ 


\title{
Sophie Albert, «Ensemble ou par pieces». Guiron le Courtois (XIII $-X V^{\mathrm{e}}$ siècles): la cohérence en question
}

\author{
Maria Colombo Timelli
}

\section{RÉFÉRENCE}

SOPHIE ALBERT, «Ensemble ou par pieces». Guiron le Courtois (XIII ${ }^{\mathrm{e}} \mathrm{XV}$ e siècles): la cohérence en question, Paris, Honoré Champion, 2010 («Nouvelle Bibliothèque du Moyen Âge» 98), pp. 642.

1 L'étiquette «Guiron le Courtois», comme on le sait, s'applique à une vaste nébuleuse de textes dont la composition s'échelonne entre 1235 environ et le début $d u \mathrm{xVI}^{\mathrm{e}}$ siècle, centrés autour de quelques héros arthuriens, beaucoup moins connus que les protagonistes d'autres sommes médiévales tels Lancelot ou Tristan, et qui appartiennent à la génération «des pères»; c'est en effet du nom de quelques-uns de ces héros - Palamedes, Meliadus, Guiron - que l'ensemble a pu prendre le titre, et ce dès les plus anciens manuscrits.

2 Sophie Albert publie ici une étude globale du «Guiron», d'après sa thèse de Doctorat (Sorbonne, 2008), étude courageuse, non seulement parce qu'elle exige la connaissance et la maîtrise d'un grand ensemble de récits, mais encore parce que ces œuvres demeurent pour la plupart inédites (un projet d'édition critique est en cours, mené par une équipe internationale que dirigent Lino Leonardi et Richard Trachsler). L'analyse s'appuie sur les deux pièces majeures de ce que l'on peut considérer comme la version de base, à savoir Meliadus et Guiron (le lecteur en trouvera le résumé en annexe, pp. 555-572), dans une perspective qui prend en compte la pluralité des aspects qui concourent à former toute production médiévale: la facture des manuscrits, le niveau du texte, celui du récit; S.A. s'efforce en effet de ne jamais oublier la matérialité de la transmission (formation des codex, assemblage des cahiers et des volumes), ni les 
questions terminologiques (comment interpréter les mots utilisés par les différents copistes: livre, estoire, piece...), ni la constitution de l'œuvre tout entière, qui se fit par ajouts successifs dans la longue durée. Pour endiguer l'ampleur de cette matière, elle a choisi de se concentrer sur trois motifs portants: la temporalité, les valeurs, les guerriers; on nous pardonnera une présentation schématique, qui ne rend pas justice à la richesse de l'analyse ni à ses nuances, mais qui permettra au moins de percevoir quelques-uns des sujets traités et l'approche adoptée.

3 La première partie déjà, intitulée L'ordre des faits, donne la mesure de l'écart qui sépare les deux récits de base: Meliadus, qui remplit les blancs narratifs des récits arthuriens existants, en ancrant l'histoire aux temps du Tristan et du Lancelot en prose, et adopte un temps orienté, linéaire, contre Guiron, qui constitue une sorte de long récit «entre parenthèses» (p. 84), où les liens temporels avec la matière de Bretagne apparaissent flous et où s'affirme plutôt l'autonomie des récits secondaires dans une temporalité orientée vers le futur. On se doute que la soudure nécessaire entre ces deux «romans» s'avère difficile: les débuts (prologues et récits liminaires) ainsi que les clôtures (explicits plus ou moins ouverts, mort ou disparition de personnages) révèlent une structure qui cumule des pièces de provenance diverse, dans un souci d'exhaustivité plus que d'unité.

4 La deuxième partie, L'ordre des valeurs, confirme l'altérité réciproque des deux romans, malgré quelques traits communs (évacuation de la mystique du Graal et de la passion d'amour): dans Meliadus les valeurs adoptées - féodales et lignagères - sont résolument celles de la cité des hommes; dans Guiron les valeurs masculines priment, le compagnonnage avant tout: l'amitié prévaut sur l'amour et même sur les liens du sang, et la critique de la femme voire la misogynie s'affichent.

5 L'ordre des guerriers, où "guerriers» désigne tous les personnages exerçant le métier des armes, est au centre de la troisième et dernière section. Deux motifs sont envisagés: la vengeance, condition nécessaire au maintien de la dignité guerrière, répondant à des contraintes reconnaissables; et les coutumes, rattachées à Uterpendragon (dans Meliadus) et à Galehaut le Brun (dans Guiron).

6 La conclusion générale rappelle comment la distinction entre les deux textes de base est confirmée tant par l'histoire des manuscrits que par les voies narratives adoptées (motifs, épisodes, valeurs sous-jacentes).

7 Parmi les compléments signalons le tableau récapitulatif des fragments de Guiron édités jusqu'à aujourd'hui (pp. 547-549), une bibliographie imposante (pp. 575-620) et deux index (personnages, pp. 621-632; manuscrits et imprimés, pp. 633-635).

8 L'analyse menée par S.A. est riche, précise, menée avec scrupule et attention: on en aura un aperçu convaincant rien qu'en parcourant la table des matières; s'il est un reproche à mouvoir, mais ce serait se montrer sévère à l'égard d'un effort dont on a souligné les mérites et les risques, il concernerait l'organisation rigide de l'examen mené et la fragmentation des résultats: conclusions d'un chapitre, d'une partie, puis conclusion générale répètent souvent les mêmes considérations, ce qui est la preuve de la validité des résultats obtenus, mais crée la sensation d'un certain nombre de répétitions.

9 En fermant ce beau livre, l'impression reste qu'on s'est approché d'une synthèse sur Guiron le Courtois, mais qu'on en est encore relativement loin: cela doit certainement être imputé, plutôt qu'à l'auteure courageuse qui a signé ces pages, à la matière même 
du livre, une matière vaste et changeante, dont - après les vastes études d'ensemble dues ces toutes dernières années à Barbara Wahlen (L'Écriture à rebours. Le Roman de Meliadus $d u$ XIII $^{e}$ au XVIII siècle, Genève, 2010), Nicola Morato (Il ciclo di Guiron le Courtois. Strutture e testi nella tradizione manoscritta, Firenze, 2011), et ici à Sophie Albert - on n'aura pas encore dit le dernier mot. 